\title{
ELECTRICAL INSULATING MATERIALS
}

\begin{abstract}
A SYMPOSIUM on the subject of electrical insulation, held at the Institution of Electrical Engineers during March 16-18, provided a very interesting picture of the vast amount of research work that has been, and is being, done on this subject throughout Great Britain in all the laboratories associated in ono way or anothor with the electrical industry. It is generally realized in the industry that the limitations of existing insulating materials constitute a formidable barrier to progress in many of its branches: voltage-gradients and working temperatures must always be kept well below the limits at which the risk of breakdown becomes appreciable, and great elaboration of design is sometimes necessary to ensure that the essential characteristics of the dielectrics employed are not adversely affected by temperature and humidity. Engineers are therefore continually investigating the processes involved in the normal operation of dielectrics and those in which breakdown occurs; they are no less concerned with the possibilities of new materials and methods of estimating their efficacy and durability under service conditions. Some thirty-two original investigations covering all these aspects of the problem came under review during the five sessions of the symposium. Consideration of the details is not possible in a short article; but a few of the points of more general scientific interest will give some idea of the present position in this field of work.
\end{abstract}

\section{Permittivity and Power Loss}

The first topic considered was the nature of the processes involved in the normal operation of insulating materials of the types used in industry : that is to say, the processes characterized by permittivity (or dielectric constant) and loss angle, the phase difference between the electric displacement within the material and the applied electric field that produces it. The importance of these quantities to the electrical engineer lies in the part that they play in determining the voltage distribution (within an insulating system), the corresponding current distribution, the precise relation between these two distributions (that is to say, the impedance, phase angle and power factor of the system) and also the temperature distribution in the state of thermal equilibrium attained under normal working conditions. The voltage-gradients and impedances (or the corresponding admittances and capacitances) are, of course, almost completely determined by permittivity alone, but the loss angle, although negligibly small for the best dielectries, is the dominant factor in determining the power dissipated within the material and is therefore of great importance in connexion with thermal equilibrium. The product of permittivity and loss angle, or strictly speaking its tangent, is proportional to the energy dissipated within the specified material during each cycle of the applied voltage. Even the smallest loss angles therefore tend to become significant when the number of cycles per second is of the order of, say, $10^{10}$, which is about the upper limit of the electrical range of frequencies - the centimetre and millimetre waves. In this region, electrical and optical techniques merge into one another, and it was appropriate therefore that, in one paper, Dr. W. Culshaw (Telecommunications Research Establishment) described a spectromoter method by which permittivity and loss angle can be measured for flat sheet material traversed by millimetre waves. The method has been shown to be practicable but has not yet yielded data beyond the reach of the established electrical methods which were employed by the other contributors-bridge methods for the lowest frequencies, circuit-resonance methods for the main radio range, and cavity resonators for centimetre waves.

The data for most of the materials of interest to engineers are of great complexity, and we are still far from a full understanding of the processes involved. Tho main characteristics of the simplest ones are, of course, well known. Part of the electric displacement is always such as can be visualized as a displacement of the electrons with respect to the atomic nuclei, a process having a natural frequency in the optical range and capable of accounting for the optical index of refraction of the material. If no additional process occurred at electrical frequencies, the dielectric constant would be equal to the square of the optical refractive index and the loss angle would be zero, since this fast displacement follows the slow electrical cycles with no appreciable lag. This condition was shown by Dr. L. Hartshorn, J. V. L. Parry and E. Rushton (National Physical Laboratory) to hold remarkably closely for the pure hydrocarbon plastics and the fluorocarbon polytetrafluorethylene. Any loss angle observed in such materials in practice can only arise from accidental impurities. The permittivity of such materials is independent of frequency but diminishes with rise of temperature by an amount corresponding with the linear expansion of the material.

Most other materials show a permittivity considerably greater than the square of the optical refractive index and therefore call for a consideration of two other displacement processes. The first is the displacement of different atomic nuclei with respect to one another, a process which may be expected to have a natural frequency in the infra-red regionthat is to say, still far enough above the electrical frequencies to give rise to no appreciable loss angle in electrical work. The second additional displacement is that arising from changes in the orientation of molecules or groups of atoms having a permanent dipole moment, changes which are characterized by a time-constant or time of relaxation rather than a frequency of vibration. The extent to which this displacement occurs, during each half-cycle of the applied field, depends on the relative values of the time constant and the duration of the half-cycle; but at frequencies for which the two are of the same order, the permittivity increases rapidly as the frequency falls and so gives the dipole orientation appreciably more time to develop, while at the same time the loss angle rises to a maximum value, which again diminishes to zero when the cycle becomes so slow that the dipole orientation follows the field without appreciable lag. The relaxation time is strongly dependent on temperature, and therefore both permittivity and loss angle are liable to vary greatly with temperature as well as frequency, and 
there is a necessary connexion between the variation of the two quantities. A paper by A. C. Lynch (Post Office Research Station, Dollis Hill) discussed this question in its bearing on the temperature coefficient of mica capacitors, emphasizing that the temperature coefficient for radio uses is liable to be quite different from that for low frequencies. The time constants of the electric displacements within the material may not in this instance arise from dipolar molecules; they may arise from differences in the distribution of resistivity and permittivity in a composite dielectric in accordance with classical theory as developed by Maxwell, but since such time constants are also temperature-dependent the same considerations apply. Dr. A. Fairweather and E. J. Frost, also of Dollis Hill, applied this simple theory to semi-conducting aggregates such as the ferrites, used primarily for their magnetic properties, though their dielectric properties are not without some influence on their performance in communication circuits. The dielectric properties, though complicated, were shown to be adequately accounted for by a network of capacitors and resistors each corresponding to some simple recognizable feature of the composite material.

Hartshorn, Parry and Rushton were particularly concerned with the dipolar process in the silicones and in representative plastics. All the long-chain silicones show a peak in the curve for loss angle at frequencies between $10^{10}$ and $10^{11} \mathrm{c} . / \mathrm{s}$. , and it is inferred that the corresponding relaxation time, which is independent of chain-length, is characteristic of the $\mathrm{Si}-\mathrm{O}$ linkage in flexible linear chains. The permittivity and loss angle of the water-absorbent plastics have been shown to be very largely dominated by the absorbed water ; but it is striking that the values for the completely dried materials, though smaller, are of the same general character as those for which the water is responsible. In other words, the absorbed water molecules behave in the electric field almost exactly like the polar groups in the structure of the pure resin, and not like free water. The main features of the observed properties are found to correspond well with a model given by Fröhlich as an extension of Debye's theory.

A. Turney (Imperial College of Science and Technology, London) reported an attempt to find evidence for a resonance process suggested by Fröhlich as also associated with the dipoles, by examining benzene derivatives having a structure considered to be most favourable for such a process; but the evidence was negative, the above-mentioned model accounting for all the observations.

\section{Liquids}

A session devoted to liquids brought out the fact that in British electrical practice the one liquid that is of supreme importance is mineral oil, transformer oil and the like. Broadly speaking, it can be regarded as a paraffin oil, initially pure but subject to contamination in use. Its primary function is to exclude moisture and air from solid insulation, usually cellulose in the form of paper or pressboard, which supplies the required mechanical properties, but only behaves as a good dielectric so long as moisture or damp air are denied access to it. The papers and discussion clearly reflected the great importance in heavy electrical engineering of the paper and oil combination, and the effect on it of moisture and other contaminants. J. Dunkley and Dr. R. W. Sillars (Metropolitan Vickers Electrical Co., Ltd.) showed that, when the oil is very highly refined, far beyond the ordinary commercial limit, it shows no measurable loss angle even when damp air is blown through it. If, however, it contains a trace of an ester, which itself produces no loss angle at low frequencies, it develops a comparatively large loss angle when allowed access to damp air. The power loss is shown to be associated with ionic conduction; the conductivity varies with the applied field because of the effect of the field on the balance between the dissociation and recombination of ions, and with the frequency because the relaxation time of this process is comparable with the periodic time of the applied voltage.

In practice the oil commonly rises in temperature to a value near $100^{\circ} \mathrm{C}$., one of its functions being to promote cooling by convection, and the high temperature with some access to air introduces further complications of great practical importance. K. $\mathrm{H}$. Stark reported an investigation carried out by the Electrical Research Association into the behaviour of ordinary oil under these conditions. The oil, when at $20^{\circ} \mathrm{C}$. or thereabouts, normally has a power factor, probably due to traces of some ionized constituent, that is very small, but accurately measurable provided the greatest possible care is taken to ensure that the capacitor in which the oil is tested is chemically clean. R. G. Martin and E. A. Patterson (Shell Petroleum Co., Ltd.) showed that the capacitor must be dismantled and the parts boiled in trichlorethylene before each test in order to ensure that the values are truly representative of the oil. Even when this is done, no unique value is obtainable for a sample of oil at $100^{\circ} \mathrm{C}$. Oxidation of the oil occurs, and Mr. Stark showed that its effect on the loss angle is a rapid diminution, which is followed by an increase. The action is accelerated by the presence of metal in the oil, probably by catalytic action at the metal surface. The quality of an oil cannot therefore be assessed by any single measurement at the working temperature.

In practice the slow continued oxidation leads to the development of acidity and conductivity in the oil, the formation of sludge, an increase in the power factor of the paper-oil mixture, and a consequent reduction of the voltage at which thermal instability would occur. Rusting of metallic iron in the system also occurs, and some of the iron compounds formed may go into solution; others may merely add to the sludge, which may ultimately become harmful by impeding the convection cooling action, but does not necessarily affect the dielectric properties to a serious extent. D. G. Childs and A. W. Stannet (British Electricity Authority) reported some interesting observations on oils that had actually been in service in various localities for periods ranging from one to twenty-four years. Simple tests of acidity and conductivity were used as an indication of condition, but conductivity did not show good correlation with acidity alone. Variations in iron content had a marked effect, and must be taken into consideration if acidity is used as a criterion of insulating quality. Their survey indicated an expectation of life of thirty-five years for the oils in general use.

\section{Breakdown}

The session devoted to a consideration of electric strength and breakdown mechanisms attracted a very large audience, for the avoidance of breakdown is a vital consideration in every electrical design. The papers, particularly one by H. F. Church and C. G. Garton, of the Electrical Research Association, 
emphasized the fact that breakdown such as is liable to occur in practice is a process far more complex than those represented by permittivity and power factor or indeed than any other occurring in electrical equipment. To the casual observer the breakdown is an instantaneous catastrophic collapse of the structure of the material, and the idea that any given material will withstand indefinitely voltage gradients up to a certain critical value, characteristic of its structure and known as its electric strength, arose naturally from the first tentative studies of the phenomenon. It is now abundantly clear that, although it is possible to determine, by voltage applied under highly special conditions, limiting voltage-gradients of this kind which are indeed characteristic of the atomic structure of the materials, yet this intrinsic electric strength is of comparatively minor importance in the much larger question of breakdown under ordinary working conditions. The intrinsic breakdown strengths of all ordinary solid and liquid insulating materials are in the region of a few $\mathrm{MV} . / \mathrm{cm}$, and they vary comparatively little with temperature and from one material to another, whereas in ordinary practice breakdown commonly occurs at an apparent voltage-gradient which is no more than one-tenth of this value; moreover, the observed value has no clear relation to the structure of the material and is greatly influenced by many other variables such as temperature, duration of application of the voltage, otc. The central problem in research on electrical insulation to-day is not so much to increase, or even to explain, intrinsic electric strengths as to raise the applied voltage-gradient at which breakdown occurs under ordinary practical conditions. C. G. Garton made the point that even halving the gap between the intrinsic electric strength and the electric strength as measured under ordinary working conditions would make possible revolutionary improvements in design.

Intrinsic electric strength was, however, not neglected. A paper by J. H. Calderwood, R. Cooper and A. A. Wallace (University of Liverpool) showed that mechanical strain may increase the value for a crystal, so that the highest value obtained for a given sample is not necessarily the true one. Another paper, by Dr. T. J. Lewis (Queen Mary College, London), described a satisfactory technique for obtaining values truly characteristic of pure organic liquids.

Breakdown at the intrinsic electric strength is an electronic process which has been reasonably well explained by the work of Fröhlich and others; breakdown under ordinary working conditions is usually a complex chain of processes, of much longer duration than the intrinsic breakdown, and perhaps including an intrinsic breakdown as part of the chain but sometimes including processes that might bo described as chemical rather than electrical. Moreover, it is not a question of a well-defined chain characteristic of a material, but of a number of possible chains, the choice depending on factors the presence of which may not be suspected - for example, the presence of sub-microscopic voids or gaseous inclusions, or ions having particular chemical properties. In this state of extreme complexity, progress is perhaps best made by isolating and studying the component processes separately, and this is what many workers are doing.

For example, papers by Dr. E. Friedlander and J. R. Reed (General Electric Co., Ltd.), by W. L. Harries (University of Oxford) and by J. H. Mason
(Flectrical Research Association) were all concerned with breakdown processes which include as the dominant feature discharges in a gas enclosed within solid insulating walls. Nearly all industrial materials contain such gaseous inclusions, which may or may not be visible: the voltage-gradient within them tends to be relatively high, and the electric strength of the gas is relatively low, so that discharges occur at moderate overall voltage gradients. Such discharges do not necessarily lead to breakdown of the insulation as a whole; indeed, a spoaker claimed that bushings in which discharges had been detected. under working voltages had been known to function satisfactorily for twenty years. However, the bombardment of the walls of the enclosure by the ions carrying the discharge may produce actual erosion of the material and a local concentration of field, which may develop progressively until complete breakdown follows. Local concentrations of field within microscopic or smaller regions of the material are probably the key to the whole problem, for, if the local stress rises to the intrinsic electric strength, local breakdown necessarily occurs and there is a probability of it developing progressively or of stimulating some chemical or thermal change which develops progressively under alternating voltage. A paper by H. G. Riddlestone (Electrical Research Association) presented evidence supporting the view that the breakdown of both polythene and polystyrene under A.C. conditions involves the development of intensely local rises of temperature, though the mechanism by which it occurs in such low-loss materials is by no means obvious.

Breakdown by thermal instability is much less surprising in the oil-pressboard combination mentioned in connexion with liquids, for the power factor is greater and its increase with rise of temperature directly measurable. R. T. Rushall (British ThomsonHouston Co., Ltd.) showed that the breakdown voltage, as judged by a straightforward one-minute test, is closely associated with the measured loss factor, irrespective of varying temperature and moisture content. This clearly indicates thermal instability as setting the limit to the safe working voltage-gradient, and moreover provides a method of estimating the risk of breakdown by a measurement of permittivity and power factor at a perfectly safe voltage.

Breakdown by short voltage impulses is usually closely related to the intrinsic electric strength, and Dr. B. Salvage (W. T. Henley's Telegraph Works Co., Ltd.) showed that the observed values for cables are very much what would be expected from the known values of intrinsic electric strength and permittivity of the constituent materials.

\section{New Materials}

Comparative studies of new and old materials for specific purposes naturally attracted much attention at the symposium: for example, the various types of impregnating varnish, the newer laminated materials, materials that have been tried in rotating machines and the special ceramics available for radio uses.

In matters of fine detail and on the comparatively small scale, chemical advances have played a prominent part. Consider, for example, the impregnating varnishes; coils wound with cotton-covered wire are impregnated with varnish which is then hardened, this being an economical process for producing a structure that is mechanically rigid and well insulated 
throughout, provided the varnish has just the right penetrating, hardening and insulating qualities. $R$. Newbound and R. T. Rushall (British ThomsonHouston Co., Ltd.) and H. R. Heap (Crompton Parkinson, Ltd.) reported on this matter. It is perhaps not surprising to find that thermo-setting varnishes incorporating phenolic resins tend to provide a better mechanical job than the older oil-oxidizing varnishes, but it would have been difficult to foresee that the use of phenolic varnish would lead to less oxidation and sludge formation when the coils are operated in oil, either by more effective covering of the copper surface, which acts as a catalyst, or by some direct inhibiting action. The difficulties of devising tests for the required combination of qualities are obvious.

On the larger scale the impression left is that chemical advances have not influenced practice so much as might have been expected. Traditional materials like mica, shellac, paper and mineral oil still seem to dominate the whole picture. It is true that polyethylene, polystyrene and the new ceramics have made possible important advances in the radio and radar fields, that glass fabrics, nylon, terylene and the silicones introduce the possibility of improvements in particular directions at a cost which may not be prohibitive, and these and many similar matters received due consideration; but no contributor was found to suggest that any revolution in practice on the large scale is in sight.

Nevertheless, the symposium as a whole gave an encouraging picture of activity and progress on a very wide front ; the many widely scattered workers thus brought together for the first time appreciated its stimulating effect, and the industry cannot fail to benefit in due course as the ideas and knowledge assembled become more widely disseminated throughout the main body of its members.

\section{HARTSHORN}

\section{ORGANIC MANURES AND FERTILIZERS}

IN the Section of Comparative Medicine of the Royal Society of Medicine a discussion was held on May 20 on "Organic Manures and Fertilizers and the Production and Composition of Food for Man and Animals". The president of the Section, Prof. C. L. Oakley, was in the chair and introduced the speakers.

Sir William Ogg (Rothamsted), who opened the discussion, outlined the present position and dealt with the soil aspects of the question. In order to maintain or increase fertility it has, from very early times, been the practice of farmers in most parts of the world to return to the soil, crop residues, animal manure and domestic waste and to rest the land for periods under grass. If production is not too intensive, this system is usually quite successful provided there is not a deficiency of some essential mineral nutrient. With increasing population and its concentration in towns, new problems have arisen. Through modern systems of sanitation, much of the manurial residue which used to find its way back to the land is now carried as sewage to the sea. The introduction of fertilizers just over a century ago enabled farmers to make good these losses and also to increase production. They have not superseded farmyard manure, which, in addition to its manurial value, has important physical effects on the soil ; but they are a source of additional plant food and without them the present population of the world could not be fed. It has been estimated that, in terms of readily available nutrients, the total amount of farmyard manure made annually in Great Britain provides only about a third of the nitrogen, a quarter of the phosphate and half the potash applied in the form of fertilizers. Fertilizer consumption has practically trebled in Britain and the United States in the past fifteen years and this has been a major factor in increasing food production.

Attempts have been made to create a controversy over the respective merits of organic manures and fertilizers. The conflict of opinion is, in fact, between those who advocate a proper use of both and a very small minority who oppose the use of fertilizers. It is sometimes alleged that fertilizers poison the soil and are injurious to the health of plants, animals and human beings. There is no sound evidence to support these views, provided that fertilizers are properly used. They do no harm to the earthworms or to the bacteria and other micro-organisms; nor do they bring about soil erosion. There is no evidence that they render plants more liable to suffer from insect, fungus or virus attacks. Organic manures and fertilizers are complementary, and in good farming practice both are employed.

Dr. G. C. Ainsworth (University College, Exeter), as a botanist and microbiologist, directed attention to the complexity of the total situation which determines health and disease in plants and of which the soil-plant interaction is only a part. Green plants are not dependent on the soil for their carbon, but they are for their other nutrients. Under natural conditions, plants die and decay in situ and so repay their debt to the soil together with interest in the form of organic matter. On cultivated land, man periodically removes a crop, and this inevitably leads to a depletion of the soil which must be made good. In primitive societies the exhausted soil is abandoned -an impossible procedure in more advanced and densely populated countries, where a balanced use of chemical fertilizers would appear to be the rational and most convenient way of maintaining the nitrogen, phosphorus and potassium, that trinity of elements basic for plant growth. Chemicals, too, are an appropriate source of the small quantities of boron, copper or other 'trace elements' which may render an unsatisfactory soil fertile.

An agricultural soil has not the simplicity of a controlled and mechanically irrigated sand culture. The organic matter it contains has a vital role in conserving moisture and in providing a substrate for the growth of micro-organisms which, in their turn, affect the physical condition of the soil by determining crumb structure and in making available materials which can be utilized by higher plants. At the same time, the microflora competes with the higher plants for nutrients, and certain fungi may cause disease, while another class, the mycorrhizal fungi, set up a symbiotic relationship with higher plants. The use of farmyard manure is the age-old solution to the problem of maintaining both the inorganic and organic constituents of the soil and the microbial population, but the advent of the internal combustion engine has rendered this solution inadequate and new sources of organic manures are having to be explored. Sometimes it is necessary to introduce the requisite symbiont for a particular plant when the plant is grown in a new situation. Finally, the summation of the soil-plant interaction, as manifest 